\title{
A SUB-PICOSECOND PULSED 5 MeV ELECTRON BEAM SYSTEM*
}

\author{
J. Paul Farrell and Ken Batchelor \\ Brookhaven Technology Group, Setauket, NY
}

I. Meshkovsky, I. Pavlishin, V. Lekomstev, A. Dyublov, and M. Inochkin Optoel Scientific Innovation Company, St. Petersburg, Russia

T. Srinivasan-Rao and John Smedley

Brookhaven National Laboratory, Upton, NY 11973

"Work supported in part by U.S. Department of Energy: Contract No. DE-AC02-98CH10886. 


\title{
A Sub-Picosecond Pulsed 5 MeV Electron Beam System
}

\author{
J. Paul Farrell ${ }^{1}$, K. Batchelor ${ }^{1}$ \\ I. Meshkovsky ${ }^{2}$, I. Pavlishin ${ }^{2}$, V. Lekomtsev ${ }^{2}$, A. Dyublov ${ }^{2}$, M. Inochkin ${ }^{2}$, \\ T. Srinivasan-Rao ${ }^{3}$ and John Smedley ${ }^{3}$ \\ ${ }^{1}$ Brookhaven Technology Group, Inc, ${ }^{2}$ Optoel Scientific Innovation Company, ${ }^{3}$ Brookhaven National Laboratory
}

\begin{abstract}
Laser excited pulsed, electron beam systems that operate at energies from $1 \mathrm{MeV}$ up to $5 \mathrm{MeV}$ and pulse width from 0.1 to 100 ps are described. The systems consist of a high voltage pulser and a coaxial laser triggered gas or liquid spark gap. The spark gap discharges into a pulse forming line designed to produce and maintain a flat voltage pulse for 1 ns duration on the cathode of a photodiode. A synchronized laser is used to illuminate the photocathode with a laser pulse to produce an electron beam with very high brightness, short duration and current at or near the space charge limit. Operation of the system is described and preliminary test measurements of voltages, synchronization and jitter are presented for a $5 \mathrm{MeV}$ system. Applications in chemistry, and accelerator research are briefly discussed.
\end{abstract}

\section{INTRODUCTION}

Picosecond and sub-picosecond pulsed electron beams have current applications in accelerator research and in pulsed radiography for the study of transient phenomena using both direct electrons and $\mathrm{x}$-rays produced by Bremsstrahlung. In accelerator research, fast pulsed high voltage systems are used to study field emission, photoemission in the presence of high fields and the formation and propagation of high brightness electron beams. Naturally, fast pulsed beams have practical use in pulse radiography where the requirements include precise synchronization between energy impulse and probe.

The first compact laser triggered sub-nanosecond high voltage generators were developed for Brookhaven National Laboratory by the Russian company, Optoel, in $1994^{1}$. They provided, respectively, a high voltage output of $0.2-0.5 \mathrm{MV}$ on a $20 \mathrm{Ohm}$ load, and $0.5-1 \mathrm{MV}$ pulse on an $80 \mathrm{Ohm}$ load with 0.1 to $0.15 \mathrm{~ns}$ rise time and pulse duration adjustable from $0.2 \mathrm{~ns}$ to $2 \mathrm{~ns}$ with $1 \mathrm{~Hz}$ repetition rate. The basic components of the generator are a low voltage LC circuit, a pulse transformer and a pulse forming line. There is a laser triggered $\mathrm{SF}_{6}$ gas switch on the output of the transformer. Sensors are located at different distances along the pulse forming line to monitor the voltage wave form.
In this paper, we describe a new, short pulse, electron beam system that uses a pulse generator, a laser triggered spark gap and very high gradient photocathode to produce a compact, short pulse synchronizable electron beam. Preliminary test results are shown for a $5 \mathrm{MeV}, 10$ to 100 ps system.

\section{THE BASIC SYSTEM}

The basic components of the short pulsed electron beam system shown in Figure 1 are:

- master timer and laser system

- high voltage pulse power supply and laser triggered spark gap

- $\quad$ pulse forming system (PFL)

- photodiode electron gun and beam transport

- diagnostic and/or experimental test region.

The master timer and laser systems are standard commercial components. The high voltage power supply consists of a pre-pulser that provides an $80 \mathrm{kV}$ pulse to the trigger electrode of a commercial spark gap. The spark gap discharges a capacitor to energize the primary coil of a high voltage resonant transformer. The output of this resonant transformer is 
developed across a coaxial high voltage spark gap. The spark gap is triggered by an axial laser pulse that discharges the high voltage output of the transformer into a pulse forming line (PFL). In the PFL, the high voltage pulse is shaped and transformed to produce a flat top high voltage of $\sim 200$ ps rise and fall time and $1 \mathrm{~ns}$ duration on the cathode of a photodiode.

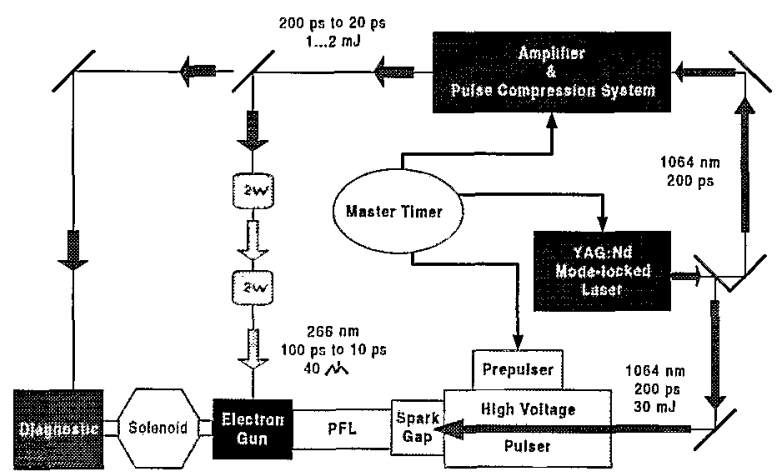

FIGURE 1. In this example, a mode locked YAG laser system (1064 nm) with $200 \mathrm{ps}$ duration is used to trigger the spark gap and the same laser is used to provide a short pulse excitation to the photocathode. The photo pulse is frequency quadrupled to obtain the optimum uv frequency range ( 255 to $266 \mathrm{~nm}$ ) for photo emission. Other laser arrangements are possible that can provide electron beam pulses down to 100 fs.

\section{Laser System}

The laser system performs three tasks:

- trigger high voltage spark gap

- illumination of the photo-cathode.

- synchronization of voltage, current and diagnostic pulse

Because the same laser provides illumination of the photocathode and the diagnostic signal, the electron beam and the probe are very highly synchronized.

\section{High Voltage Pulse Power Supply}

The high voltage pulse power supply consists of the low voltage pulse generator, a resonant pulse transformer, capacitance and a laser triggered spark gap switch. The purpose of the high voltage pulse power supply is to produce a voltage pulse on the high-voltage electrode of the laser triggered spark gap switch.
In operation, a low voltage pulse system generates voltage pulses with amplitude $25-100 \mathrm{kV}$ on the primary coil of the resonant transformer. Standard commercial spark switches are used as switching elements in this part of the circuit. An $80 \mathrm{kV}$ prepulser is used to trigger this spark gap. The duration of the first half wave voltage on the primary coil of the pulse transformer is $\sim 700 \mathrm{~ns}$. Transformer oil is used as insulating medium for both the low voltage pulser and the resonant pulse transformer.

On the 5 MV pulser, the resonant pulse transformer is designed to produce an output voltage of up to $2.5 \mathrm{MV}$ (1st half wave) with pulse duration 500 ns. The transformer consists of four main parts: the casing, the primary coil, high-voltage winding and high-voltage capacitance. The total capacitance of high-voltage electrode and high-voltage capacitance to ground is $\sim 60 \mathrm{pF}$.

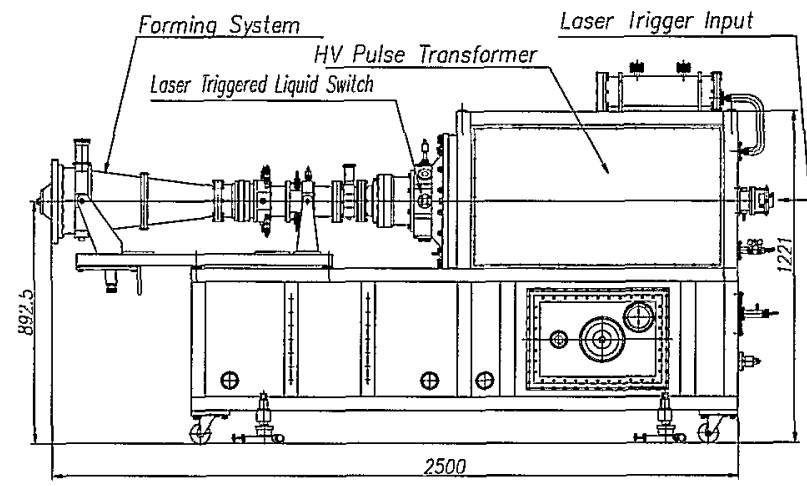

FIGURE 2. Side view of the $5 \mathrm{MV}$ high voltage pulser.

The dielectric material in the output high voltage laser triggered spark gap is pressurized $\mathrm{SF}_{6}$ gas for low voltage systems. A special dielectric liquid is used in the $5 \mathrm{MV}$ system. The purpose of the laser triggered spark gap is to switch the energy accumulated at the output of the pulse transformer and to form a rectangular pulse with minimum time spread due to commutation of the spark gap. A coaxial tube with glass rod passes through the volume of the highvoltage pulse transformer and serves to transport the laser pulse to the inter-electrode gap.

\section{Pulse Forming System (PFL)}

The purpose of the pulse forming system is to produce an output pulse with rise time of $\sim 150 \mathrm{ps}$ and duration of $-1 \mathrm{~ns}$ at the photocathode. The pulse forming system of the $5 \mathrm{MV}$ pulser consists of five parts: the charging inductance, the forming line, a selfbreakdown switch, a transport line and the voltage 
transforming section. The impedance of the line seen at the triggered spark gap is $10 \mathrm{Ohms}$ and the pulse length is $1 \mathrm{~ns}$. This impedance determines the duration of the pulse at the output of the PFL. The rise time and jitter of the pulse at the cathode are dependent on reliable and consistent operation of a low-inductance, multi-channel, self-breakdown liquid switch that is incorporated in the PFL The final output voltage of 5 MV is achieved at the cathode by cylindrically symmetric transformation from an initial $10 \mathrm{Ohm}$ line to final impedance of $160 \mathrm{Ohm}$. The output of the transforming line is terminated in a characteristic resistive impedance at the vacuum diode.

Preliminary tests of the operation of the $5 \mathrm{MV}$ pulser are currently underway. First the liquid switch was tested with $25 \mathrm{~mm}$ gap. The liquid switch was triggered with use of the Nd-YAG laser at a wave length of $1060 \mathrm{~nm}$, pulse duration of $200 \mathrm{ps}$ and pulse energy of $10-30 \mathrm{~mJ}$. The laser pulse was transported along the axis of the pulse transformer and into the dielectric switch from the side of the negative electrode. The applied voltage was $2.5 \mathrm{MV}$. At this voltage, the starting delay was measured to be $20-25$ ns and the jitter was $0.5 \mathrm{~ns}$.

Next, the $10 \mathrm{Ohm}$ pulse forming line was connected to the high-voltage transformer and its performance was tested. The choice of recharging inductance enabled the line to fully charge in $3-5 \mathrm{~ns}$ with multiplication coefficient of $1.2-1.3$ with respect to the transformer output voltage.

Finally, the tapered impedance transforming section of the PFL was tested. In this section, the impedance terminating impedance is $160 \mathrm{Ohms}$ which results in a factor of four increase in voltage. Capacitive probes used to measure voltage along the PFL were calibrated by driving the sections at low voltage using a solid state pulser with $\sim 150 \mathrm{ps}$ rise time.

\section{The Photo-Diode Electron Gun}

The electron gun is a photodiode with a copper cathode and $1 \mathrm{~mm}$ stainless steel aperture ${ }^{2-6}$. The anode-cathode gap can be adjusted in-situ so the cathode can be conditioned without exposing the surface to atmosphere. To produce high brightness beams, the electric field at the cathode is $\sim 1 \mathrm{GV} / \mathrm{m}$. With a $1 \mathrm{~mm}$ diameter laser spot at the cathode, the steady state space charge limited emission current is $500 \mathrm{~A}$. After conditioning, the dark current is less than $1 \%$ of the photocurrent.
Beam current higher than the space charge limit can be achieved with short pulse operation but internal pressure due to Coulomb forces causes longitudinal and transverse expansion of the beam.

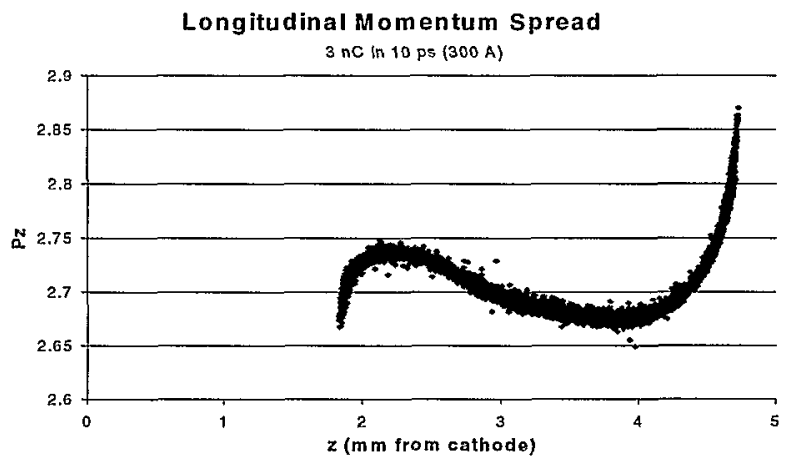

FIGURE 3. Longitudinal momentum spread for a $3 \mathrm{nC}, 10$ ps bunch calculated using the time dependent PIC code, MAFIA. $P_{z}$ is in units of $\beta \gamma$.

Figure 3 shows the spatial pulse length and momentum spread of a $1 \mathrm{MeV}$ beam at a distance of 3 $\mathrm{mm}$ from the cathode $(\sim 2 \mathrm{~mm}$ beyond the anode). Coulomb forces have pushed the leading edge of the bunch toward higher energy while edge is retarded in energy. As the beam progresses, the bunch width expands due to the difference in energy of the leading and trailing ends.

\section{Beam Transport and Synchronization}

The electron bunch is accelerated to relativistic speed in a distance of less than $1 \mathrm{~mm}$ in the photodiode. This rapid acceleration reduces effects of space charge and results in a beam with low divergence and small diameter at the anode. After exiting from the high field region of the diode, the beam diverges due to the lens effect of the transition from high field to no field. A solenoid lens is placed up close to the anode to collect the beam and focus it prior to injection into another high energy accelerator or for to the low energy diagnostic tests or experiments.

The $5 \mathrm{MeV}$ system is designed to operate continuously at a pulse repetition rate of $0.1 \mathrm{~Hz}$. The pulse rate is limited by the power of the charging system. Higher repetition rate is possible with larger power supplies and it may be necessary a heat exchanger to remove heat from the low voltage pulser. 


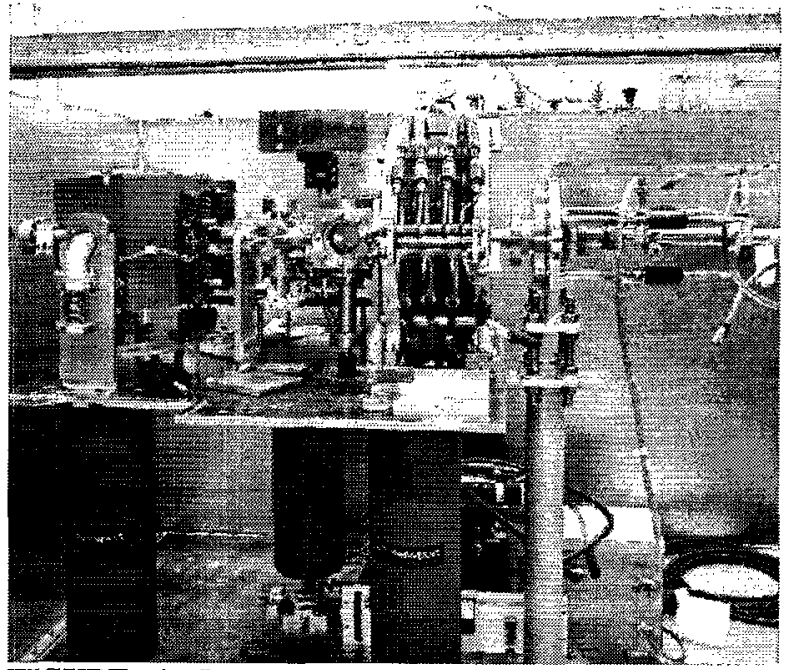

FIGURE 4. Beam transport system at BNL used to characterize the beam from the sub-picosecond electron gun. The cathode is just above the stanchion on the right. It is followed by a solenoid focusing magnet and a dipole magnet that is used to measure energy spread.

When the high voltage laser trigger pulse, the laser pulse to the photodiode and the laser diagnostic (probe) pulse are derived from the same laser system synchronization depends primarily on the jitter in the high voltage spark gap. This jitter has been measured to be $<\sim 0,5 \mathrm{~ns}$. Jitter in the firing of the high voltage spark gap affects the energy of the pulse as well as the arrival time of the flat pulse at the cathode.

The synchronization of arrival of the electron bunch and the diagnostic pulse is simplified because it depends only on the difference in path length between the two pulses. This single laser method can be used down to $10 \mathrm{ps}$ in duration. For applications that require electron bunches less than $10 \mathrm{ps}$, it will be necessary to employ a two laser system.

\section{CONCLUSION}

We have built and are currently testing a new 5 $\mathrm{MeV}$ pulsed electron beam system that is designed to produce electron bunches in the $10 \mathrm{ps}$ to $100 \mathrm{ps}$ range. The system can be configured to produce shorter bunches $(\sim 100 \mathrm{fs})$ using a more complex, and more expensive, two laser system.

Applications include accelerator research and use as an injector into higher voltage accelerators. It can also be used as a complete system for the study of transient phenomena using direct electrons or Bremsstrahlung.

\section{ACKNOWLEDGMENTS}

We would like to acknowledge the work of $\mathrm{J}$. Smedley for doing the MAFIA calculations and for measurements of quantum efficiency in the presence of high fields and we would like to acknowledge Thomas Tsang, John Schill and Robert Conde for keeping the laser and high voltage pulser systems operating during the installation and characterization runs. This work is supported in part by DOE SBIR DE-FG02-97ER82336. The work at BNL is supported by DOE contract number DEACO2-98CH10886

\section{REFERENCES}

1. T. Srinivasan-Rao and J. Smedley; Table Top, Pulsed, Relativistic Electron Gun with GV/m Gradient; BNL 63517; pres. 7th Advanced Accelerator Concepts Workshop, Lake Tahoe, CA, 12-18 October (1996);AIP Conf. Proc. 398, eds. S. Chaltopadhyay, J. Mc Cullough, \& P. Dahl, p. 730, AIP, Woodbury, NY (1997).

2. K. Batchelor, J. P. Farrell, G. Dudnikova, I. Ben-Zvi, T. Srinivasan-Rao, J. Smedley, and V. Yakimenko; A High Current, High Gradient, Laser Excited, Pulsed Electron Gun; BNL 65678 ; pres. EPAC'98, Stockholm, Sweden, 22-26 June (1998); Proc. 6th European Particle Accelerator Conf., S. Myers, L. Liljeby, Ch. Petit-JeanGenaz, J. Peole, \& K.-G. Rensfelt, eds., p. 791 (1998).

3. T. Stinivasan-Rao, J. Smedley, K. Batchelor, J. P. Farrell, and G. Dudnikova; Comparison of Electrostatic and Time Dependent Simulation Codes for Modeling a Pulsed Power Gun; BNL 65748 ; pres. 12th Int'l. Conf. on High Power Particle Beams (Beams'98), Haifa, Israel, 7-12 June (1998); M. Markovits and J. Shiloh, eds., IEEE Catalog \#98EX103, p. 549 (1998).

4. T. Srinivasan-Rao, J. Smedley, J. Schill, K. Batchelor, and J. P. Farrell; Dark Current Measurements at Field Gradients Above $1 \mathrm{GM} / \mathrm{m}$; BNL 65746; pres. 8th Workshop on Advanced Accelerator Concepts, Baltimore, MD, 6-11 July (1998).

5. T. Srinivasan-Rao, J. Smedley, K. Batchelor, J. P. Farrell, and G. Dudnikova; Optimization of Gun Parameters for a Pulsed Power Electron Gun; BNL 65748 ; pres. 8th Workshop on Advanced Accelerator Concepts, Baltimore, MD, 6-11 July (1998);

6. T. Srinivasan-Rao, J. Schill, I. Ben-Zvi, K. Batchelor, J.P. Farrell, J. Smedley, X.E. Lin, and A. Odian; Simulation, Generation, and Characterization of High Brightness Electron Source at $1 \mathrm{GV} / \mathrm{m}$ Gradient; BNL 66464; pres. PAC'99 Conf., New York, NY, 3/29-4/2/99; Proc. 1999 Particle Accelerator Conf., Eds. A. Luccio \& W. Mackay, p. 75 (1999). 